\title{
Algoritmo Rápido para Cálculo de Centroide de Imagem com Sensores Digitais de Sol do tipo CMOS para aplicação em Pequenos Satélites
}

\author{
Diego A. Coutinho, Fernando F. Ramborger, Antonio A. Ferreira Junior e Evandro C. Vilas Boas
}

\begin{abstract}
Resumo-Esse trabalho apresenta um algoritmo simples e rápido para cálculo de centroide de imagem com sensores digitais de Sol do tipo CMOS (complementary metal-oxide semiconductor). Interpreta-se a imagem como uma matriz de pixel e soma-se as intensidades de linhas e colunas, armazenando-as em vetores. Obtém-se as coordenadas do centroide $\left(x_{c}, y_{c}\right)$ verificando o índice correspondente ao valor de maior intensidade do vetor linha e coluna, respectivamente. Comparou-se essa abordagem ao algoritmo básico para cálculo de centroide com thresholding em termos de tempo de processamento com redução da ordem de $58 \%$ e precisão similar.
\end{abstract}

Palavras-Chave-Algoritmo, centroide de imagem, CMOS, sensor digital de Sol.

Abstract-This work presents a fast image centroiding algorithm for CMOS-based digital sun sensors. The image is interpreted as a pixel matrix. The rows and columns pixels intensities are summed and stored in vectors. The centroid coordinates $\left(x_{c}, y_{c}\right)$ are obtained by checking the row and column vector index-related higher pixel intensity. This approach was compared to the basic centroiding and thresholding method with a time processing reduction of $\mathbf{5 8 \%}$ and similar precision.

Keywords - CMOS-based sun sensor, image centroiding algorithm, digital sun sensor.

\section{INTRODUÇÃO}

Sistemas de determinação e controle de atitude são responsáveis por determinar o sentido e a direção em que um determinado satélite se encontra. Assim como, controlar a movimentação do satélite de modo a prover o posicionamento alvo em relação à um referencial [1-3]. Nesse processo, podese empregar, em conjunto, sensores e atuadores. Os sensores subdividem-se em inerciais e não-inerciais. O giroscópio e o acelerômetro classificam-se como sensores de inércia, relacionando-se respectivamente à velocidade angular e à aceleração. Os sensores não-inerciais determinam a posição angular do satélite em relação à um referencial. Integram essa classe de sensores o sensor de Sol, magnetômetro, sensor de estrela e o sensor de Terra. Os atuadores se diferenciam quanto ao tipo de torque em externo (bobinas de torque magnético e propulsores) e interno (rodas de reação e giroscópio de momentum).

Diego A. Coutinho, Fernando F. Ramborger, Antonio A. Ferreira Junior e Evandro C. Vilas Boas, Laboratório de Cyber Segurança e Internet das Coisas (CS\&I Lab.), Instituto Nacional de Telecomunicações - Inatel, Santa Rita do Sapucaí1, e-mail: diego.anestor@gec.inatel.br, fernandoramborger@gec.inatel.br, antonioa@inatel.br, evandro.cesar@inatel.br. Este trabalho foi parcialmente financiado pela Fundação Instituto Nacional de Telecomunicações - Finatel.
Os sensores de Sol permitem obter o vetor de orientação do satélite (atitude) em relação ao Sol ou qualquer outra fonte de luz que simule o Sol em um ambiente controlado [46]. Utilizam-se relações trigonométricas para determinar os ângulos de rotação, conhecidos como ângulos de Euler, dos eixos do plano cartesiano do satélite em relação ao Sol. Dessa forma, pode-se orientar e manter o satélite inerte em uma posição previamente estabelecida por meio dos atuadores. Os sensores de Sol destacam-se por apresentar baixa complexidade e custo de projeto, hardware compacto e baixo consumo de energia, sendo amplamente utilizados em pequenos satélites [2]. Classificam-se esses sensores quanto à tecnologia em analógicos e digitais.

Os sensores digitais geralmente utilizam sensores de imagem CMOS (complementary metal-oxide semiconductor) para a captura de imagens da radiação solar incidente [4-6]. Posiciona-se uma máscara de material opaco com uma ou múltiplas aberturas sobre o plano de detecção [4]. Os raios solares iluminam a superfície da máscara e atravessam-na por meio de abertura(s) se projetando no plano de detecção. Forma-se uma imagem do Sol, cujo centroide permite determinar os ângulos de Euler do satélite em relação ao Sol. Para isso, processa-se a imagem por meio de algoritmos para determinar com precisão as coordenadas do centroide.

Existem alguns algoritmos utilizados para o cálculo dessas coordenadas [4-6, 8-10]. Dentre eles, destaca-se o método básico para cálculo de centroide (basic centroiding method, $\mathrm{BCM}$ ), que relaciona a intensidade dos pixel da imagem. Esse método pode proporcionar maior índice de erro devido à presença de ruido na intensidade dos pixel durante a formação da imagem. Logo, empregam-se algumas técnicas de préprocessamento da imagem para reduzir o ruído e prover maior precisão no cálculo do centroide. Dentre essas técnicas, citamse o uso de limiar (thresholding method, TM) e a filtragem de imagem (image filtering method, IFM). O TM estabelece um determinado valor de intensidade como limitante para redefinir a intensidade do pixel. Se o valor for superior ao limiar preserva-se ou atribui-se um segundo valor de intensidade pré-definido. Caso contrário, considera-se a intensidade do pixel igual a zero. Essa técnica sujeita-se a menor ou maior precisão de acordo com o limiar estabelecido. Por outro lado, a filtragem implementa técnicas para remover ou mitigar o ruído relacionando a intensidade do pixel com a de seus vizinhos. Outras abordagens consideram o uso de imagens modelo e figuras geométricas como forma de reduzir o tempo de cálculo das coordenadas do centroide da imagem [5, 9]. 


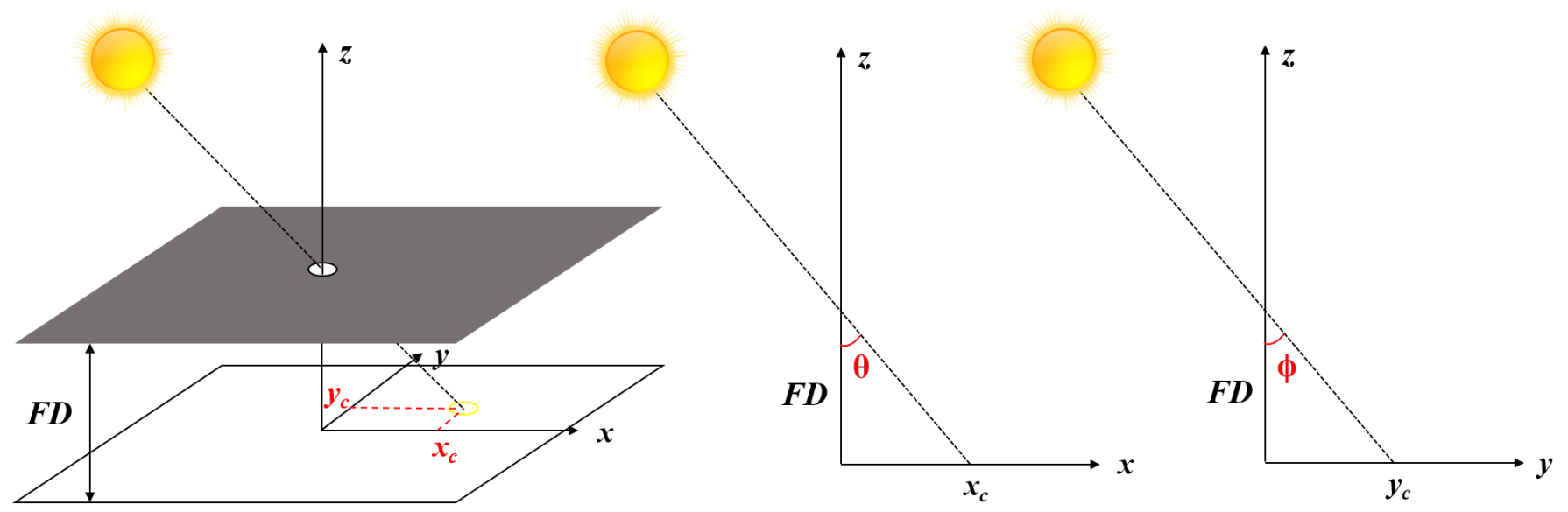

Fig. 1. Princípio de operação de sensores de Sol digitais e relações trigonométricas para cálculo de centroide de imagem.

Esse trabalho propõe uma nova abordagem para o cálculo do centroide que processa diretamente a imagem sem a necessidade de empregar técnicas de pré-processamento como as supracitas. Obtém-se menor tempo no cálculo das coordenadas do centroide em relação ao algoritmo básico e ao uso do método de limiar (basic centroiding thresholding mehtod, BCTM) com precisão similar. Organiza-se o trabalho em cinco seções. Na Seção II, discute-se o princípio de operação de sensores digitais de Sol e explicita-se o algoritmo BCTM. Apresentase a nova abordagem e compara-a as técnicas supracitadas em termos de precisão na Seção III. Na Seção IV, avaliam-se o tempo de processamento e cálculo dos ângulos de Euler, considerando o algoritmo BCTM e o proposto nesse trabalho. Discutem-se as principais conclusões e elencam-se trabalhos futuros na Seção V.

\section{PRINCÍPIO DE OPERAÇÃo DE SENSOR DigITAL DE SOL}

Os sensores digitais de Sol integram basicamente uma máscara de material opaco e um plano de detecção [4-9]. O plano de detecção corresponde a matriz de pixel que forma a imagem da luz solar. A máscara pode conter abertura única ou múltiplas de acordo com a abordagem no cálculo e determinação das coordenadas do centroide [4]. Tem-se máscaras de abertura única e circular comumente implementada em pequenos satélites. Assim como, propostas de máscaras com múltiplas aberturas circulares. Verificam-se aberturas em formato de fendas perpendiculares posicionadas próximas ao centro ou a borda da máscara [10-12]. Esse trabalho limita-se aos sensores digitais de Sol com máscara de abertura circular única.

Nesse tipo de sensor, uma parcela dos raios solares que iluminam a superfície da máscara atravessa a abertura circular central e projeta-se sobre uma determinada região do plano de detecção com maior intensidade, como visto na Fig. 1. Por conseguinte, forma-se uma imagem da projeção da luz do Sol sobre o plano de detecção, cujas coordenadas do centroide $\left(x_{c}\right.$, $\left.y_{c}\right)$ permitem encontrar os ângulos $(\theta, \phi)$ de incidência dos raios solares em relação aos planos $x$ e $y$, respectivamente. Entre o plano da máscara e o plano de detecção, tem-se uma distância focal $(F D)$. Dessa forma, aplicam-se relações trigonométricas para obter $\theta$ e $\phi$, conforme indicado na Fig. 1:

$$
\begin{aligned}
& \theta=\arctan \left(\frac{x_{c}}{F D}\right) \\
& \phi=\arctan \left(\frac{y_{c}}{F D}\right)
\end{aligned}
$$

Em (1) e (2), considerou-se o eixo das coordenadas centralizado em relação ao plano de detecção. Por meio do cálculo desses ângulos, o sistema de determinação e controle de atitude pode ser realimentado para prover a correta orientação do satélite através dos atuadores. Portanto, torna-se necessário obter as coordenadas do centroide com boa precisão. O algoritmo básico para processamento da imagem e cálculo do centroide (BCM) é dado por [4-6]:

$$
\begin{aligned}
& x_{c}=\frac{\sum_{i=1}^{N} x_{i} I_{i}}{\sum_{i=1}^{N} I_{i}} \\
& y_{c}=\frac{\sum_{=1}^{N} y_{j} I_{j}}{\sum_{i=j}^{N} I_{j}}
\end{aligned}
$$

sendo $x_{i}$ e $y_{j}$ a posição de cada pixel na matriz e $I$ a intensidade dos pixel da imagem. A precisão no cálculo desses valores relaciona-se ao quão bem definida encontra-se a imagem do centroide. Para esse trabalho, empregou-se um sensor de imagem com pixel de dimensão $0,0016 \mathrm{~mm}$ e distância focal $(F D)$ igual à $2,3 \mathrm{~mm}$. Dessa forma, o erro de um pixel no cálculo do centroide implica em um erro de 0,0398 graus na determinação de $\theta$ e $\phi$. Ao realizar o pré-processamento de imagem por meio de um limiar para redefinição dos valores dos pixels, pode-se obter maior precisão no cálculo do centroide por meio de (3) e (4). Na Fig. 2, exemplificase o pré-processamento da imagem por meio do método de limiar, comparando-o a imagem originalmente capturada pelo sensor. Verifica-se melhor definição da projeção da luz do Sol. Dado que considerou-se zero a intensidade dos pixel que inicialmente estavam abaixo do limiar. 

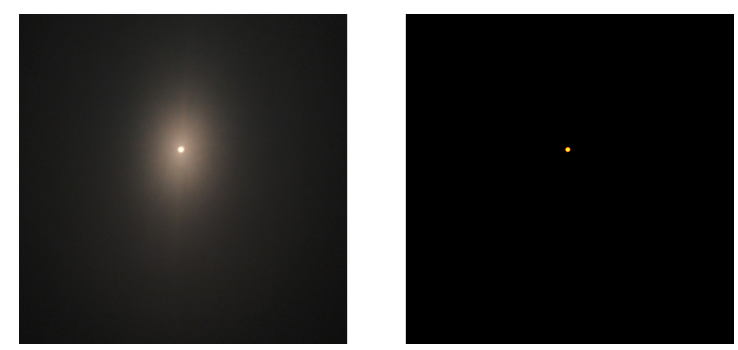

Fig. 2. Imagem capturada pelo sensor e tratada utilizando método de limiar.

\section{Algoritmo PixelMax}

Nessa seção propõe-se uma nova abordagem para simplificar e reduzir o tempo de cálculo das coordenadas do centroide da imagem capturada pelo sensor, além de eliminar a necessidade de pré-processamento. Compara-se esse algoritmo ao BCTM.

\section{A. Algoritmo PixelMax}

O algoritmo PixelMax interpreta a imagem como uma matriz de pixel e acumula as intensidades de cada linha e coluna, armazenando-os em dois vetores distintos, como visto na Fig. 3. Em seguida, avalia o maior valor armazenado e a respectiva posição do vetor que representa os índices de posição do pixel centroide. Em linhas gerais, o algoritmo realiza os seguintes passos:

- Passo 01: Importar a imagem e gerar a matriz de pixel;

- Passo 02: Acumular simultaneamente as intensidades linha a linha e coluna a coluna e armazenar em dois vetores distintos;

- Passo 03: Verificar o maior valor cumulativo do vetor linha obtido no passo 02 e extrair o índice da linha do pixel centroide;

- Passo 04: Verificar o maior valor cumulativo do vetor coluna obtido no passo 02 e extrair o índice da coluna do pixel centroide;

- Passo 05: Utilizar os índices para buscar o pixel centroide e determinar as respectivas coordenadas do centroide;

- Passo 06: Determinar os ângulos $\theta$ e $\phi$ por meio das relações em (1) e (2);

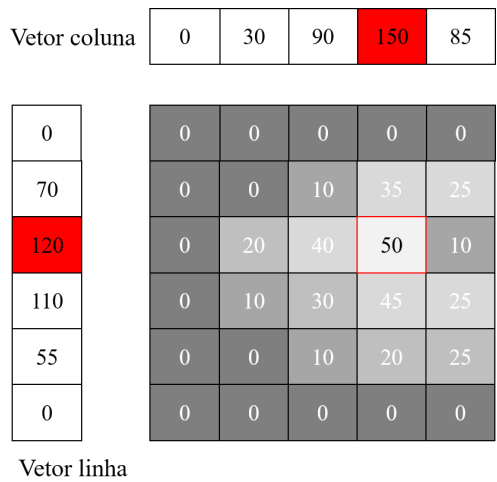

Fig. 3. Ilustração do algoritmo proposto.

Aplicou-se o algoritmo em duas imagens capturadas de uma fonte luminosa posicionada sobre o sensor de imagem em diferentes posições. Na Fig. 4, verifica-se a imagem e o ponto obtido pelo algoritmo em verde. Para facilitar a visualização, empregou-se um quadrado com vários pixels em tonalidade verde. Contudo, a extremidade superior esquerda desse quadrado é a indicação do pixel centroide. Verifica-se bom grau de precisão no cálculo do centroide por meio da abordagem proposta.
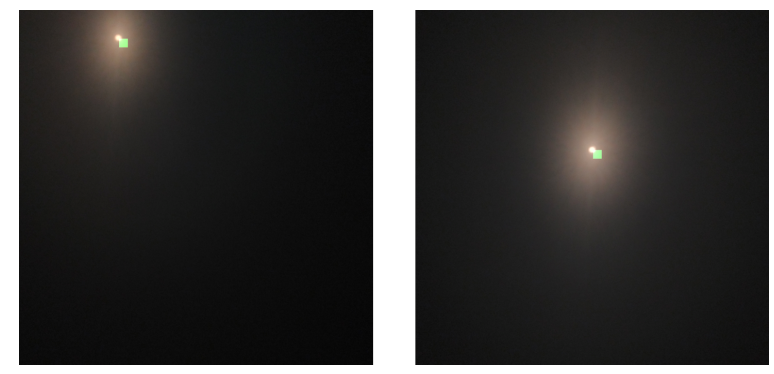

Fig. 4. Cálculo do centroide pelo algoritmo PixelMax.

\section{B. Comparação com o algoritmo BCTM}

Para fins de comparação em termos de precisão, aplicou-se o algoritmo PixelMax e o algoritmo básico para cálculo de centroide em um conjunto de quatro imagens distintas, como visto na Fig. 5.
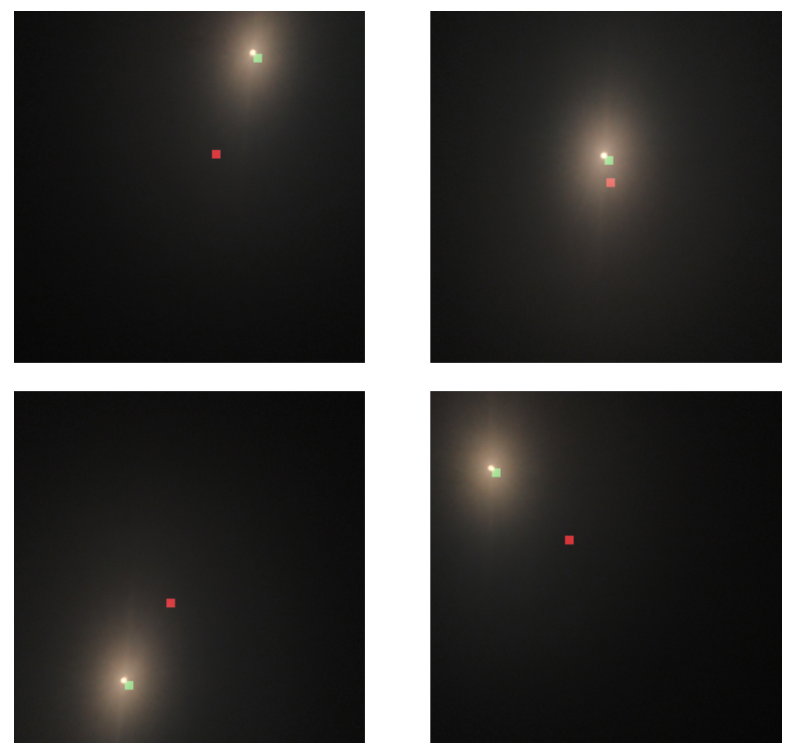

Fig. 5. Cálculo do centroide pelos algoritmos BCM (quadrado vermelho) e PixelMax (quadrado verde).

Nota-se que o algoritmo BCM (quadrado vermelho) apresenta erro considerável no cálculo das coordenadas do centroide, acentuados para posições distante do centro da imagem. Por outro lado, o algoritmo proposto garante a assertividade em diferentes casos. Considerando o pré-processamento da imagem por meio do método de limiar, observa-se que a abordagem em (3) e (4) converge para valores similares ao obtido pelo algoritmo proposto, como pode ser visto na Fig. 6. Para fins de comparação visual, projetou-se o resultado para o algoritmo PixelMax (Fig 5) em conjunto com o resultado obtido para o algoritmo básico após o pré-processamento. 

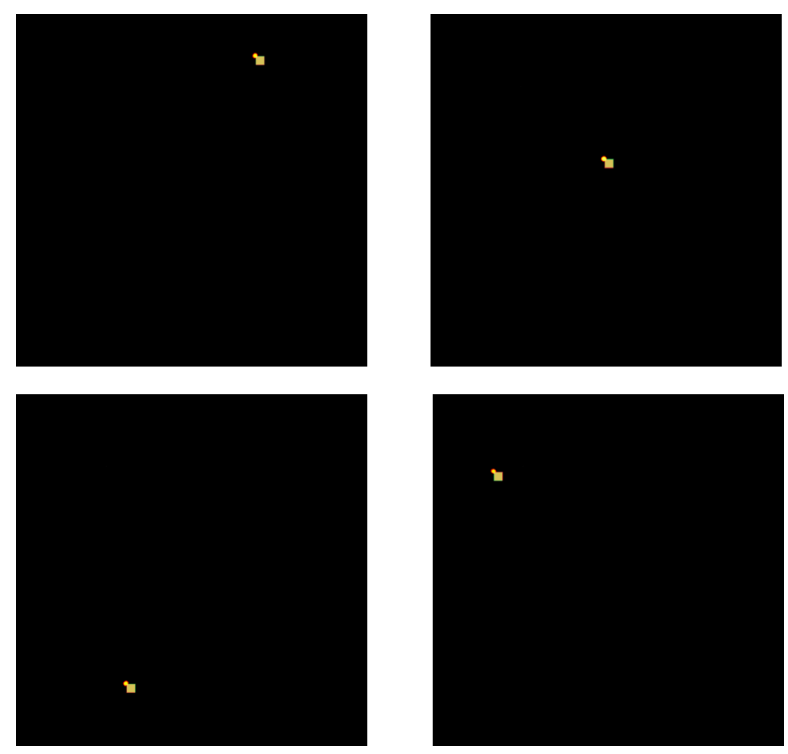

Fig. 6. Cálculo do centroide pelos algoritmos BCTM (quadrado vermelho) PixelMax (quadrado verde) sem pré-processamento.

\section{ANÁlise de DESEMPENHO E COMPARAÇÃo DE PRECISÃO DOS ALGORITMOS}

Nessa seção, apresenta-se uma análise de desempenho entre o algoritmo BCTM e PixelMax em termos de tempo de processamento computacional e precisão. Para isso, capturaram-se um conjunto de trinta imagens por meio do sensor de imagem. Além disso, estruturam-se os códigos em linguagem Python de modo que apenas as linhas de códigos dos algoritmos supracitados sejam diferentes. Dessa forma, tem-se o mesmo conjunto de comandos para importação e interpretação da imagem, assim como para o cálculo dos ângulos de Euler. Executaramse os códigos cinco vezes para o mesmo conjunto de imagens, resultando em 150 interações ao todo. Utilizou-se a biblioteca cProfile do Python para obter os tempos de execução total e médio das interações. Na Tabela I, apresentam-se os tempos de execução de ambos os códigos.

\section{TABELA I}

TEMPO TOTAL E MÉDIO (S) DE PROCESSAMENTO DOS ALGORITMOS.

\begin{tabular}{|c|c|c|}
\hline Algoritmo & Tempo total (s) & Tempo médio (s) \\
\hline BCTM & 1658,748 & 11,058 \\
\hline PixelMax & 689,564 & 4,597 \\
\hline
\end{tabular}

Observa que o algoritmo proposto reduz o tempo de processamento e cálculo dos ângulos de Euler em cerca de $58 \%$, enquanto mantém assertividade em relação ao algoritmo BCTM, como visto na Tabela II por meio da comparação dos valores de $\theta$ e $\phi$. Além disso, obteve-se simplicidade e facilidade de implementação do algoritmo.

\section{CONClusões}

Esse trabalho propôs um algoritmo simples e rápido para cálculo das coordenadas do centroide de imagens capturadas por sensores digitais de Sol do tipo CMOS (complementary metal-oxide semiconductor), denominado PixelMax. Esse algoritmo interpreta a imagem como uma matriz de pixel e soma as intensidades de linhas e colunas, armazenando-as em vetores. Posteriormente, determinam-se as coordenadas do centroide verificando o índice correspondente ao valor de maior intensidade do vetor linha e coluna, respectivamente. Comparou-se essa abordagem ao método básico para cálculo de centroide com pré-processamento de imagem (basic centroiding thresholdingmehtod, BCTM). O algoritmo PixelMax proveu redução de cerca de $58 \%$ no tempo de processamento e cálculo dos ângulos de Euler com precisão próxima ao algoritmo BCTM. Trabalhos futuros compreendem a evolução do algoritmo para reduzir o número de pixel processados e otimizar o tempo de resposta do algoritmo, preservando a simplicidade e facilidade de implementação. Além disso, visase realizar um estudo de precisão do algoritmo em ambiente controlado, como explicitado em [4], e comparar os resultados com o algoritmo BCTM.

TABELA II

ÂNGULOS $\theta$ E $\phi$ OBTIDOS POR MEIO DOS ALGORITMOS.

\begin{tabular}{|c|c|c|c|c|}
\hline \multirow{2}{*}{ Imagem } & \multicolumn{2}{|c|}{ Algoritmo BCTM } & \multicolumn{2}{c|}{ Algoritmo PixelMax } \\
\cline { 2 - 5 } & $\theta$ & $\phi$ & $\theta$ & $\phi$ \\
\hline 1 & $-17,125$ & $-30,787$ & $-17,328$ & $-30,846$ \\
\hline 2 & 0,203 & $-8,398$ & 0,219 & $-8,253$ \\
\hline 3 & $-6,667$ & 20,401 & $-6,607$ & 20,501 \\
\hline 4 & $-27,423$ & 9,999 & $-27,349$ & 10,001 \\
\hline
\end{tabular}

\section{AgRAdECIMENTOS}

Os autores agradecem a Fundação Instituto Nacional de Telecomunicações (Finatel) por fomentar esse trabalho e ao Instituto Nacional de Telecomunicações (Inatel) por prover os meios necessários à sua realização.

\section{REFERÊNCIAS}

[1] S. Ikari et al., "Attitude Determination and Control System for the Procyon Micro-Spacecraft,"Transactions of the Japan Society for Aeronautical and Space Sciences, vol. 60, no. 3, pp. 181-191, 2017.

[2] M.J. Sidi, Spacecraft Dynamics Control, Cambridge University Press, Cambridge, 1997.

[3] Y Yoo, "Attitude Control System Design Verification for CNUSAIL1 with Solar/Drag Sail,"International Journal of Aeronautical \& Space Sciences, vol. 17, no. 4, 579-592, 2016.

[4] G. Rufino e M. Grassi, "Multi-Aperture CMOS Sun Sensor for Microsatellite Attitude Determination,"Sensors, vol. 9, pp. 4503-4524, 2009.

[5] Y.-K. Chang e B.-H. Lee, "Development of high-accuracy image centroiding algorithm for CMOS-based digital sun sensors,"Sensors and Actuators A, vol. 144, no. 27, pp. 29-37, 2008.

[6] M. Buonoucore, M. Grassi e G. Rufino, "Aps-basedminiature sun sensor for earth observation nanosatellites,"Acta Astronautica, vol. 56, pp. 139$145,2005$.

[7] W.K. Pratt, Digital Image Processing, John Wiley and Sons, New York, 1991.

[8] H. Zhu, S. Sun e "Sun Sensor Based on CMOS APS,", Infrared Technology, vol. 26, no. 4, pp. 76-80, 2004.

[9] L. He e Y. Hu, "A fast algorithm of attitude measurement for APS-based digital sun sensors,"in 2006 1st International Symposium on Systems and Control in Aerospace and Astronautics, 2006, pp. 39-41, 2006.

[10] R. Saleem, S. Lee e J. Kim, "A cost-effective micro sun sensor based on black sun effect,"in 2017 IEEE SENSORS, pp. 1-3, 2017.

[11] Ł. Farian et al., "A Miniaturized Two-Axis Ultra Low Latency and Low-Power Sun Sensor for Attitude Determination of Micro Space Probes,"IEEE Transactions on Circuits and Systems I: Regular Papers, vol. 65, no. 5, pp. 1543-1554, 2018.

[12] C. Jing, T. Liang e Q. Lei, "A new kind of miniature sun sensor design,"2016 IEEE International Conference on Electronic Information and Communication Technology (ICEICT), pp. 219-222, 2016. 